\title{
Application of the Oxidation of Hydrogen Peroxide for DNA Sensing Based on Platinum Deposition
}

\author{
Tomoyuki Yasukawa*, Riku Moede and Fumio Mizutani \\ Graduate School of Material Science, University of Hyogo, \\ 3-2-1 Kouto, Kamigori, Ako, Hyogo 678-1297, Japan
}

(Received February 2, 2015; accepted May 21, 2015)

Key words: disposable screen-printed carbon electrode, platinum deposition, hybridization, intercalation, hydrogen peroxide

An electrochemical detection system for DNA has been investigated based on the current increase due to the catalytic oxidation of hydrogen peroxide with platinum deposited by the electrochemical reduction of chloro-2,2':6',2"-terpyridine platinum (II) chloride dihydrate (Pt complex) on a screen-printed carbon electrode. The platinum deposited on the screen-printed carbon electrode that has shown no catalytic activity for oxidation of hydrogen peroxide gives rise to catalytic activity. Cyclic voltammetry was used to reduce the Pt complex to deposit platinum metal on the carbon electrode. The oxidation current of hydrogen peroxide increased with increasing concentration of the $\mathrm{Pt}$ complex in the electrolytic deposition. The intercalation of the Pt complex into doublestranded DNA (dsDNA) decreased the concentration of free Pt complex and caused a decrease in the diffusion coefficient of the intercalated Pt complex. Moreover, the reduction of the Pt complex was inhibited due to steric hindrance. Thus, the oxidation current for hydrogen peroxide by platinum deposited on the carbon electrode decreased with an increase in the concentration of dsDNA. This procedure is absolutely simple without the need to immobilize DNA. Furthermore, the use of inexpensive screenprinted carbon electrodes will allow for the development of disposable sensing systems.

\section{Introduction}

Electrochemical DNA biosensors have been studied to develop a simple, sensitive, and inexpensive platform for gene identification for human genetic diseases, early diagnosis of infectious diseases promoted by pathogens, and screening of novel drug candidates. The detection process of single-stranded DNA (ssDNA) targets with specific sequences consists mainly of two important steps: the recognition of target ssDNA by

*Corresponding author: e-mail: yasu@sci.u-hyogo.co.jp 
the capture ssDNA by hybridization, and the transduction of the hybridization event to an electric signal. Interaction between double-stranded DNA (dsDNA) formed by hybridization and electrochemical probe molecules leads to the difference in the electrochemical responses and the ability to determine the target ssDNA selectively and quantitatively.

Electrochemically active bis-intercalaters ${ }^{(1)}$ and threading intercalators, ${ }^{(2-4)}$ which readily insert into the planar moiety between complementary duplex bases, have been used to indentify hybridized target ssDNA. Detection of the shift of the redox potential after the redox species intercalate into dsDNA has also been applied to discriminate the target DNA from the DNA duplex on the electrode. ${ }^{(5)}$ Labeling ssDNA has also frequently been used to determine DNA hybridization. Metal nanoparticles captured by a DNA hybrid were detected by stripping methods ${ }^{(6-8)}$ or by the potential difference of ionselective electrodes ${ }^{(9)}$ after labeled particles were chemically dissolved by strong acid. Enzyme labels have also been used to produce signal molecules for highly sensitive electrochemical detection. ${ }^{(10-12)}$ However, ssDNA for capturing target ssDNA has to be immobilized on an electrode for the discrimination of the target ssDNA to separate the uncaptured target ssDNA.

We have previously reported the highly sensitive and simple determination of dsDNA using the electrolytic deposition of cis-diamminedichloroplatinum(II) (cisplatin) on glassy carbon (GC) electrodes. ${ }^{(13)}$ The deposition of the platinum metal on GC electrodes which have no catalytic activity for proton reduction enables catalytic proton reduction. Suppression of the platinum deposition by the complexation of cisplatin and dsDNA was used to develop the sensitive dsDNA sensors. The method is simple because the immobilization of capture DNA is unnecessary. However, the strategy does not support the determination of DNA with specific sequences. More recently, we proposed a sensitive and simple technique for the determination of a DNA sequence based on the combination of intercalation of chloro-2,2,':6',2"-terpyridine platinum (II) chloride dihydrate (Pt complex) with dsDNA formed by hybridization and the catalytic reduction of protons with the platinum deposited on GC electrodes. ${ }^{(14)}$ However, it is difficult to remove the deposited platinum completely by polishing the electrode surface, which may lead to poor reproducibility.

In this study, we employed screen-printed carbon electrodes to demonstrate dsDNA determination based on the suppression of platinum deposition based on the decrease in concentration of free Pt complex because of intercalation. The use of inexpensive screen-printed carbon electrodes allows the development of disposable sensing systems. Catalytic oxidation of hydrogen peroxide was used to determine the platinum deposition. Figure 1 shows the principle of the electrochemical determination of dsDNA. In the absence of target DNA, the Pt complex is reduced to deposit metallic platinum on screen-printed carbon electrodes, which is followed by an increase in oxidation current due to hydrogen peroxide on the deposited platinum surface [Fig. 1(a)]. The presence of dsDNA causes a decrease in the concentration of the free Pt complex because of intercalation and thereby decreases the oxidation current of hydrogen peroxide [Fig. $1(b)]$. 

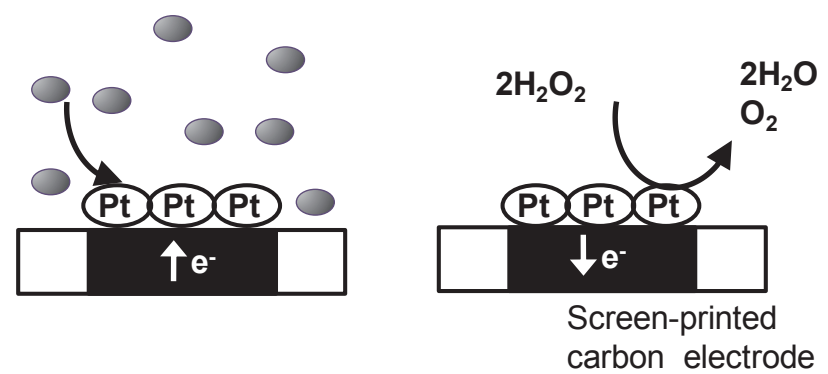

(a)
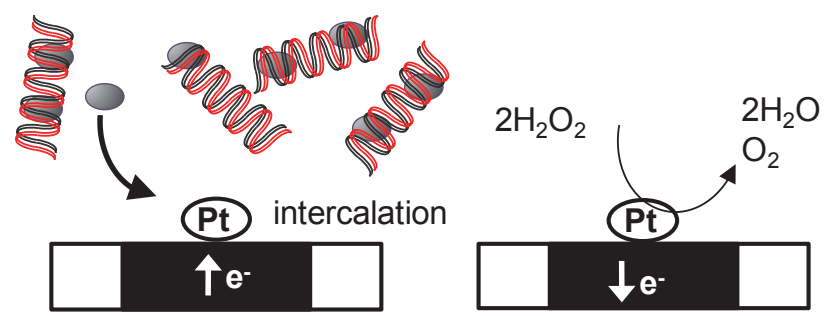

Pt complex :

dsDNA: תNaned

(b)

Fig. 1. (Color online) Principle of the electrochemical determination of dsDNA concentration by the intercalation of Pt complexes into dsDNA in (a) the absence and (b) the presence of dsDNA.

\section{Materials and Methods}

A screen-printed carbon electrode $(1.5 \mathrm{~mm}$ in diameter) which was fabricated on a polyimide film by a screen-printer (LS-150RV, Newlong Seimitsu Kogyo, Tokyo, Japan) was donated by Prof. Itagaki and Prof. Shitanda (Tokyo University of Science). Cyclic voltammetry $(\mathrm{CV})$ was performed by sweeping the potential of the screen-printed carbon electrode with a scan rate of $50 \mathrm{mV} \mathrm{s}^{-1}$ in phosphate buffer solution containing 10.0 $\mu \mathrm{M}$ Pt complex to determine the reduction potential of the Pt complex. An $\mathrm{Ag} / \mathrm{AgCl}$ electrode and coiled platinum wire were used as the reference and counter electrodes, respectively. Complexes of $\mathrm{Pt}$ were synthesized according to published procedures. ${ }^{(15)}$ Amperometric measurements were also performed with stirring to confirm the deposition of metallic platinum by reduction of the Pt complex from the increase of catalytic current due to protons.

A solution containing dsDNA refined from herring sperm ( $\leq 50 \mathrm{bp})$ was obtained from Sigma (St. Louis, MO). Different concentrations of dsDNA were added to $0.1 \mathrm{M}$ phosphate buffer ( $\mathrm{pH}$ 7.0) containing $2.5 \mu \mathrm{M}$ Pt complex to ensure the capture of the $\mathrm{Pt}$ complex by intercalation in dsDNA. After incubation for $30 \mathrm{~min}$ at room temperature, $\mathrm{CV}$ was performed to deposit the platinum on the surface of the screen-printed carbon 
electrode by the reduction of Pt complex. The potential was scanned over 10 cycles from $0.0 \mathrm{~V}$ to $-1.2 \mathrm{~V}$ with a scan rate of $50 \mathrm{mV} \mathrm{s}^{-1}$. CV was then carried out in phosphate buffer solution containing $10.0 \mathrm{mM}$ hydrogen peroxide using the screen-printed carbon electrode with deposited platinum to measure the catalytic oxidation of hydrogen peroxide by the deposited platinum.

\section{Results and Discussion}

\subsection{Reduction of platinum complex}

We investigated the reduction potential of the Pt complex on the screen-printed carbon electrode. Figure 2(a,i) shows the cyclic voltammogram of 5.0 $\mu \mathrm{M}$ Pt complex in $0.1 \mathrm{M}$ phosphate buffer ( $\mathrm{pH} 7.0)$. The reduction current was observed to be below -1.0 $\mathrm{V}$ and increased greatly beyond $-1.1 \mathrm{~V}$ in the presence of Pt complex [Fig. 2(a,i)], while no reduction response was observed in the absence of Pt complex [Fig. 2(a,ii)]. After the electrode was washed with phosphate buffer, the CV was recorded in phosphate buffer without the Pt complex. The reduction response obtained without the Pt complex was similar to that obtained in the presence of Pt complex in Fig. 2(a,i). Therefore, the results suggest that the Pt complex was reduced to deposit metallic platinum on the screenprinted carbon electrode below $-1.0 \mathrm{~V}$, which follows the large increase in the reduction current below $-1.1 \mathrm{~V}$ due to proton and oxygen reduction on the deposited platinum

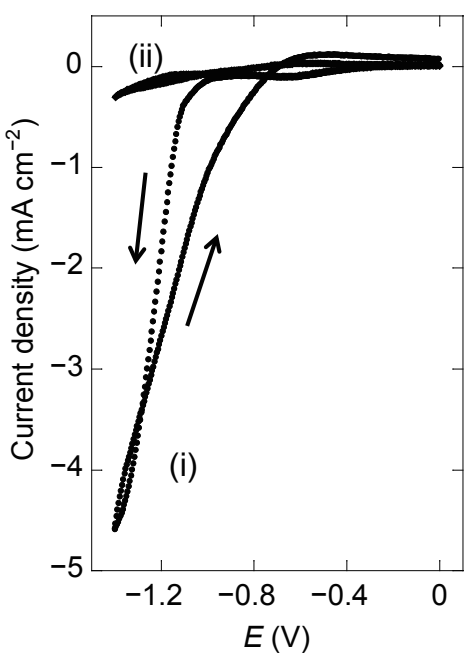

(a)

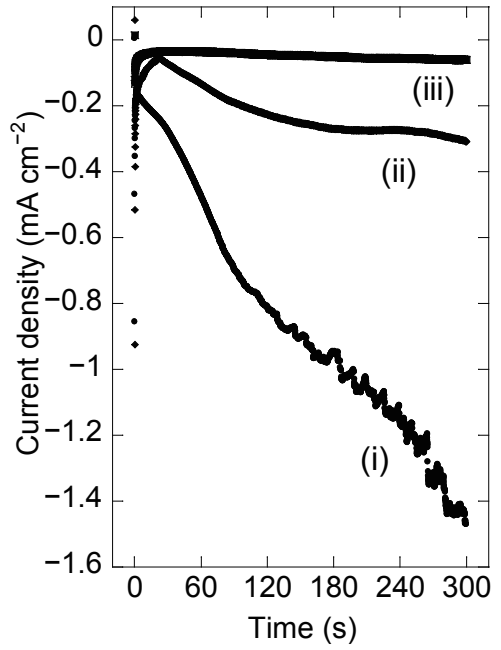

(b)

Fig. 2. (a) Cyclic voltammograms obtained by the screen-printed carbon electrodes (i) in the presence and (ii) in the absence of $5.0 \mu \mathrm{M}$ Pt complexes. (b) Amperograms obtained in the presence of $5.0 \mu \mathrm{M} \mathrm{Pt}$ complexes. The potentials of screen-printed carbon electrodes were stepped down from $0.4 \mathrm{~V}$ to (i) -1.10 , (ii) -1.05 , and (iii) $-0.70 \mathrm{~V}$. 
surface. The small reduction currents obtained at $-0.6 \mathrm{~V}$ in the presence and absence of Pt complex could be caused by the reduction of oxygen dissolved in the buffer or the adsorption of protons.

Figure 2(b) shows amperograms obtained using screen-printed carbon electrodes in $0.1 \mathrm{M}$ phosphate buffer containing $5.0 \mu \mathrm{M}$ Pt complex. The potential was stepped down from $0.4 \mathrm{~V}$ to the different potentials at zero seconds to reduce the Pt complex. When the potentials were stepped below $-1.05 \mathrm{~V}$, reduction currents gradually increased due to proton reduction followed by platinum deposition on the screen-printed carbon electrode after a step-like response appeared [Figs. 2(b,i) and 2(b,ii)]. However, the application of $-0.70 \mathrm{~V}$ resulted in a slight steady-state current with no increase in the reduction current [Fig. 2(b,iii)]. The results also indicate that the Pt complex was reduced on the electrode at potentials below $-1.0 \mathrm{~V}$. Unstable current responses were observed $120 \mathrm{~s}$ after the application of $-1.1 \mathrm{~V}$ [Fig. 2(b,i)] because of the formation of hydrogen gas on the electrodes due to the electrochemical reduction of protons. Moreover, the screen-printed carbon film was physically removed from the polyimide sheet because of the generation of hydrogen bubbles from the carbon film. Thus, amperometric measurements of the increase of the catalytic current due to protons cannot be used to investigate the interaction between the Pt complex and dsDNA.

\subsection{Catalytic oxidation of hydrogen peroxide by the screen-printed carbon electrodes with deposited platinum}

Catalytic currents were investigated by CV in phosphate buffer containing $10.0 \mathrm{mM}$ hydrogen peroxide. Figure 3 shows CVs obtained using screen-printed carbon electrodes with deposited platinum by potential sweeps of 10 cycles in the presence of different concentrations of Pt complex. Clear oxidation and reduction currents for hydrogen peroxide were observed in voltammograms obtained with the electrodes containing deposited platinum [Figs. 3(a)-3(c)]. The oxidation current for hydrogen peroxide increased with increasing concentration of Pt complex in the deposition step of platinum. A high overpotential was required to oxidize and reduce hydrogen peroxide using screen-printed carbon electrodes without deposited platinum [Fig. 3(d)]. In addition, no hydrogen bubble formation was observed during the application of the potential for 10 cycles between $0.0 \mathrm{~V}$ to $-1.2 \mathrm{~V}$. Therefore, measurement of the catalytic oxidation current of hydrogen peroxide by the deposited platinum is suitable for determining the concentration of the Pt complex.

\subsection{Effect of the addition of dsDNA}

The appearance of a catalytic reduction current of hydrogen peroxide was used to determine the concentration of dsDNA. The Pt complex used in this work is well known to act as an intercalator with a binding constant to dsDNA of $3.9 \times 10^{5} \mathrm{M}^{-1}$. ${ }^{(16)}$ In addition, the binding ratio was also reported to be $0.2 \mathrm{Pt}$ complex per nucleotide. ${ }^{(17)}$ Different concentrations of dsDNA from herring sperm were added to $0.1 \mathrm{M}$ phosphate buffer containing $2.5 \mu \mathrm{M}$ Pt complex. After incubation for $30 \mathrm{~min}, \mathrm{CV}$ was performed to deposit the platinum. Figure 4 shows the voltammograms obtained in buffer containing 

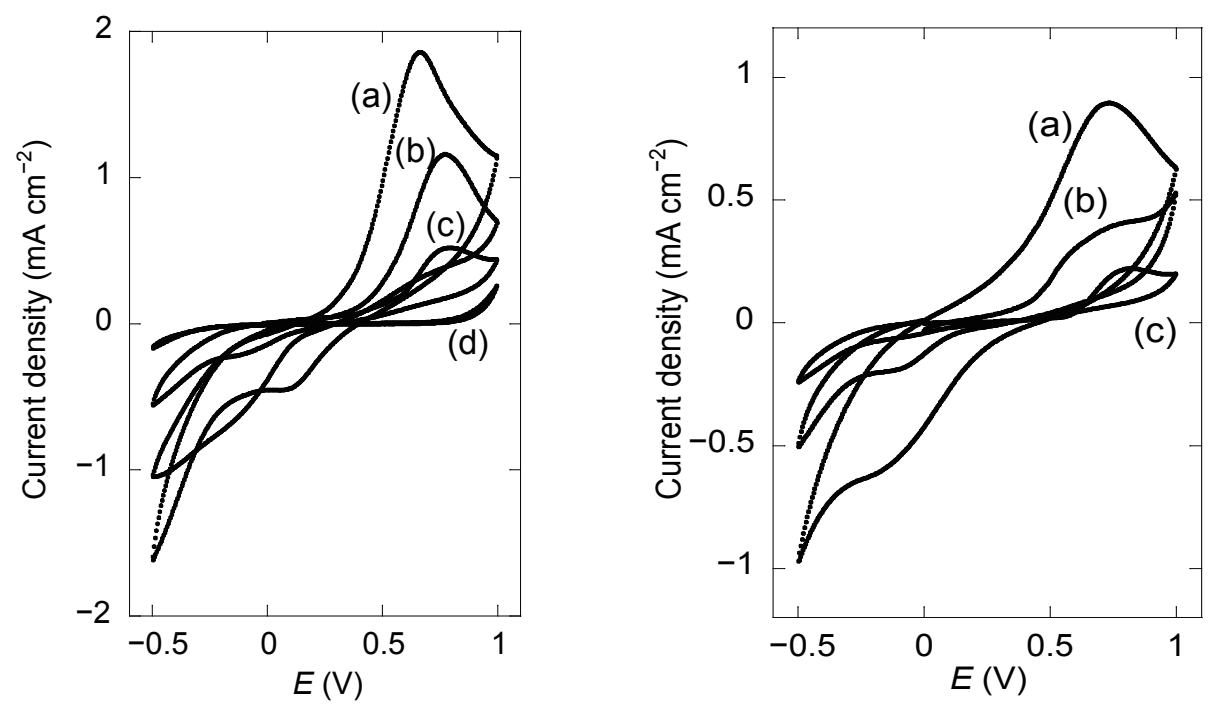

Fig. 3 (left). Cyclic voltammograms obtained in phosphate buffer containing $10.0 \mathrm{mM}$ hydrogen peroxide using screen-printed carbon electrodes with deposited platinum by potential sweeps of 10 cycles in the presence of (a) 5.0, (b) 2.5, (c) 1.0, and (d) $0 \mu \mathrm{M}$ of Pt complex.

Fig. 4 (right). Cyclic voltammograms obtained in buffer containing $10.0 \mathrm{mM}$ hydrogen peroxide using screen-printed carbon electrodes with deposited platinum by potential sweeps of 10 cycles in the presence of $2.5 \mu \mathrm{M}$ Pt complex and different concentration of dsDNA from herring sperm. Concentration of dsDNA: (a) 0.4 , (b) 1.0 , and (c) $2.0 \mathrm{ng} \mathrm{mL}^{-1}$.

10.0 $\mathrm{mM}$ hydrogen peroxide using electrodes with deposited platinum. The catalytic oxidation current for hydrogen peroxide decreased with increasing concentration of dsDNA. The Pt complexes intercalated into added dsDNA, leading to a decrease in both free Pt complex concentration and the amount of platinum deposited on the electrode surface. The intercalation of the Pt complex into dsDNA caused a restriction in its electrochemical reduction due to steric hindrance and an increase in its diffusion coefficient. The catalytic oxidation current is significantly reduced by increasing the dsDNA concentration up to $2.0 \mathrm{ng} \mathrm{mL}^{-1}$ [Fig. 4(c)]. A dsDNA concentration as low as 0.4 ng $\mathrm{mL}^{-1}$ can be detected in this system [Figs. 3(b) and 4(a)]. These results suggest that dsDNA concentration can be determined from the catalytic current of hydrogen peroxide detected by disposable screen-preinted carbon electrodes.

\section{Conclusions}

We propose a simple determination of dsDNA concentration based on the catalytic current for hydrogen peroxide detected after platinum deposition on screen-printed carbon electrodes. It is difficult to apply amperometric measurements of the catalytic reduction current of protons to the determination of dsDNA concentrations, because 
the carbon films peeled off with the removal of hydrogen bubbles generated by the electrochemical reduction of protons. Thus, the dsDNA concentration was estimated by the catalytic oxidation current of hydrogen peroxide. The catalytic current depended on the amount of deposited platinum, which decreased with decreasing concentration of free Pt complex due to the intercalation of Pt complex into the dsDNA. The disposable screen-printed carbon electrodes can be used for the simple determination of dsDNA concentration by mixing a Pt complex into the solution containing the targeted dsDNA.

\section{Acknowledgements}

This work was partly supported by a Grant-in-Aid for Scientific Research (No. 26288071) of The Ministry of Education, Culture, Sports, Science and Technology, Japan. The authors are grateful to Prof. Isao Shitanda and Prof. Masayuki Itagaki (Tokyo University of Science) for the fabrication of screen-printed carbon electrodes.

\section{References}

1 E. G. Hvastkovs and D. A. Buttry: Anal. Chem. 79 (2007) 6922.

2 S. Takenaka, K. Yamashita, M. Takagi, Y. Uto and H. Kondo: Anal. Chem. 72 (2000) 1334.

3 S. Takenaka, Y. Uto, H. Saita, M. Yokoyama, H. Kondo and W. D. Wilson: Chem. Commun. (1998) 1111.

4 Z. Gao and N. Tansil: Anal. Chim. Acta 636 (2009) 77.

5 E. L. S. Wong and J. J. Gooding: Anal. Chem. 78 (2006) 2138.

6 J. Wang, R. Polsky and D. Xu: Langmuir 17 (2001) 5739.

7 J. Wang, D. Xu, A.-N. Kawde and R. Polsky: Anal. Chem. 73 (2001) 5576.

8 S. Pinijsuwan, P. Rijiravanich, M. Somasundrum and W. Surareungchai: Anal. Chem. 80 (2008) 6779.

9 A. Numnuam, K. Y. Chumbimuni-Torres, Y. Xiang, R. Bash, P. Thavarungkul, P. Kanatharana, E. Pretsch, J. Wang and E. Bakker: J. Am. Chem. Soc. 130 (2008) 410.

10 F. Patolsky, A. Lichtenstein and I. Willner: Nature Biotechnol. 19 (2001) 253.

11 E. Kim, K. Kim, H. Yang, Y. Kim and J. Kwak: Anal. Chem. 75 (2003) 5665.

12 P. Fanjul-Bolado, D. Hernández-Santos, M. B. González-García and A. Costa-Garcí: Anal. Chem. 79 (2007) 5272.

13 Y. Yoshimoto, T. Yasukawa and F. Mizutani: Analyst 13 (2009) 2113.

14 T. Yasukawa, Y. Yamashita, R. Moede, D. Nakayama, S. Iijima and F. Mizutani: Analyst 140 (2015) 1014.

15 G. T. Morgan and F. H. Burstall: J. Chem. Soc. (1934) 1498.

16 L. Messori, G. Marcon, A. Innocenti, E. Gallori, M. Franchi and P. Orioli: Bioinorg. Chem. Appl. 3 (2005) 239.

17 K. W. Jennette, S. J. Lippard, G. A. Vassiliades and W. R. Bauer: Proc. Natl. Acad. Sci. U.S.A. 71 (1974) 3839. 